\title{
The impact of stochastic microstructures on the macroscopic fracture properties of brick and mortar composites
}

\author{
J. William Pro \\ Department of Mechanical Engineering, 2235C Engineering II Building, University of California, Santa Barbara, \\ CA 93106, USA \\ Rone Kwei Lim \\ Department of Computer Science, 5110 Harold Frank Hall, University of California, Santa Barbara, CA 93106, USA \\ Linda R. Petzold \\ Department of Mechanical Engineering and Department of Computer Science, 5107 Harold Frank Hall, University \\ of California, Santa Barbara, CA 93106, USA \\ Marcel Utz \\ School of Chemistry, University of Southampton, SO17 1BJ, UK \\ Matthew R. Begley ${ }^{1}$ \\ Materials Department and Department of Mechanical Engineering, 2361A Engineering II Building, University of \\ California, Santa Barbara, CA 93106, USA
}

\begin{abstract}
This paper examines the effect of non-uniform microstructures on the macroscopic fracture properties of idealized brick and mortar composites, which consist of rigid bricks bonded with elasticplastic mortar that ruptures at finite strain. A simulation tool that harnesses the parallel processing power of graphics processing units (GPUs) was used to simulate fracture in virtual specimens, whose microstructures were generated by sampling a probability distribution of brick sizes. In the simulations, crack advance is a natural outcome of local ruptures in the cohesive zones bonding the bricks: the macroscopic initiation toughness for small-scale yielding is inferred by correlating the critical load needed to advance a pre-defined crack with an associated far-field energy release rate. Quantitative connections between the statistical parameters defining heterogeneous brick distributions and the statistics of initiation toughness are presented. The nature of crack tip damage and stresses ahead of the crack tip are illustrated as a function of brick size variability. The results offer
\end{abstract}

\footnotetext{
${ }^{1}$ begley@engineering.ucsb.edu, fax:(805) 893-8486
} 
quantitative insights that can be used to identify microstructural targets for process development, notably specific brick size distributions that still provide macroscopic toughening.

\section{Introduction}

The ability to connect local microstructural features with macroscopic mechanical properties is a central challenge in materials development, as such connections effectively define 'processing targets' associated with acceptable levels of heterogeneity. This challenge is particularly acute for quasi-brittle composites that exploit ordering of microstructural features, as local disruptions to ordering can serve as defects that limit global performance [1-4]. Bio-inspired 'brick and mortar' composites are an excellent example of this [4-9]; the ideal microstructure will have perfectly aligned stiff features and achieve toughness through perfectly overlapping features that spread damage (or equivalently, provide bridging over large length scales).

However, the physical reality is that most high through-put processing routes invariably introduce heterogeneous distributions of brick size and therefore overlap distances, which limit the effectiveness of the microstructure. As such, the macroscopic performance of these composites is less than optimal, as real materials (both natural and synthetic) exhibit imperfections at different length scales, including large defects/pores [10-17], distributions in brick sizes and shape [18-20], and distributions in mortar properties (e.g. strength) from interface to interface. These features not only reduce the effective macroscopic mechanical properties of the material (broadly defined), but they can also lead to substantial variability in properties from one test specimen to the next $[5,6,21-24]$.

The stress-strain response of brick and mortar composites has been modeled extensively in a deterministic sense via analytical models as well as conventional finite element simulations $[22,25-$ 32]. However, even for uniform brick arrangements, direct links between microstructure and the resulting fracture toughness (arguably their most desirable property) are limited [6, 29, 33, 34]. It should be noted that while the work-to-failure during uniform deformation is related to toughness, the presence of non-uniform deformation at the tip of a crack leads to important differences [33]. To our knowledge, the impact of non-uniform and statistically-defined brick arrangements on macroscopic fracture toughness has not been analyzed. As a result, there is a significant need for a quantitative understanding of the link between statistical distributions in microstructure (e.g., 
brick sizes, shape, mortar properties) and the resulting statistics of fracture properties.

Previous analyses of the impact of heterogeneous brick arrangements on uni-axial behavior have provided insights that are relevant to the present explicit study of fracture toughness. These works defined representative volume elements (RVEs) of brick arrangements within finite element models with embedded cohesive zones $[18,35]$. For example, Barthelat et al. studied the effect of statistical size and shape distributions in the microstructure using a large RVE containing several hundreds of tablets generated via Voronoi tiling. Their results indicated important differences in composite stress-strain response of a microstructure with statistical variations when compared to that of a 'perfect' microstructure, and they are in excellent agreement with experiment [18]. In the work of Bekah et al., the authors also implemented statistics within the microstructure by introducing a random variation in tablet length and offset [35]; the results indicated a reduction in both strength and work to failure as a result of the variation. Moreover recently, statistical strength predictions in scaffolded ceramic structures (analogous to natural nacre) have begun to emerge [30]; these calculations have enabled predictions of strength probability distributions in periodic materials, and can be generalized for arbitrary geometry and loading.

The objective of this work is to elucidate the statistical characteristics of macroscopic fracture properties that result from statistical variations in local microstructural features, using as a reference the 'perfect' material (e.g., one with no variation in microstructure). The current models exploit the highly efficient GPU-based computational approaches developed by Pro et al. and Lim et al. $[33,34]$ to conduct a broad number of virtual fracture tests. The results confirm some of the trends suggested by previous works, yet provide more complete statistical links between parameters describing microstructural heterogeneity and macroscopic fracture properties. The simulations utilize virtual specimens comprising several hundreds of thousands of bricks, with sizes determined via sampling from a statistical distribution. The approach explicitly tracks individual motions of each brick (all of different sizes) and predicts crack evolution through the rupture of cohesive zones bonding the bricks. The efficiency of the numerical method allows a large number of virtual tests, such that the statistics of the response can be well quantified.

The results offer new insight into the design of synthetics, illustrating the role of statistics in the microstructure on both the average and standard deviation in initiation fracture toughness, as well as the resulting damage and stress distributions ahead of a dominant pre-crack. The simulation 
results give key insight into the sensitivity of the composite to local variations in brick size, in terms of the computed average and variance from a statistically significant subset of virtual fracture tests.

\section{Modeling Approach}

\subsection{Microstructural Idealizations}

As illustrated in Fig. 1, the material is modeled using an idealized microstructure consisting of an overlapping grid of comparatively stiff bricks bonded together by thin, compliant mortar sections. For many synthetic composites the bricks can be modeled as rigid bodies with only rotational and translational degrees of freedom, as described in detail in Pro et al [33]. The mortar, presumed to be present in low volume fraction, is idealized with a piece-wise cohesive zone law (shown schematically in Fig. 2) inserted between all brick faces, and is characterized by a finite stiffness $\left(k_{n}\right)$, strength $\left(\sigma_{0}\right)$, and work to failure $\left(\Gamma_{i}\right)$. Note that this cohesive zone law may be alternatively expressed in terms of the critical and full rupture displacements $\left(\Delta_{Y}\right.$ and $\Delta_{R}$, respectively, as shown in Fig. 2) of the interface, as follows: $\Delta_{Y}=\sigma_{0} / k_{n}$ and $\Delta_{R}=\Gamma_{i} / \sigma_{0}$. Naturally, this gives rise to a dimensionless interface ductility, defined as $\Delta_{R} / \Delta_{Y}$ (or alternatively as the ratio $k_{n} \Gamma_{i} / \sigma_{0}^{2}$ ), which was shown in [33] to directly scale the resolution and size of the plastic zone (in bricks). To limit the scope of the study, it is assumed that the bricks all have the same height (h), and that the mortar (interface) properties are constant from interface to interface; the impact of different brick heights and statistical mortar behaviors is left for future work.

Heterogeneity within the microstructure is introduced by assigning a random width (defined via the aspect ratio $\bar{w}_{i}=w_{i} / h$ ) to each brick in the system (dictated by sampling a skew-normal brick size distribution using computer-generated pseudo-random numbers [36]), and subsequently stacking them in an arrangement of space-filling rectangles as shown in Fig. 1d. The distribution in brick widths is defined in terms of its population mean $\langle\bar{w}\rangle$ (i.e. average brick size), standard deviation in brick size $\Delta \bar{w}$, and distribution skewness $\gamma$. The skewness is defined as follows:

$$
\gamma=\frac{\sqrt{n} \sum_{i=1}^{n}\left(\bar{w}_{i}-\langle\bar{w}\rangle\right)^{3}}{\left(\sum_{i=1}^{n} \sqrt{\left(\bar{w}_{i}-\langle\bar{w}\rangle\right)^{2}}\right)^{3}}
$$

This choice of statistical size distribution is motivated by optical imaging experiments in natural composites [18-20], which have strongly indicated the brick sizes are indeed normally distributed 
and follow no spatial correlation in the their distribution. While there are few reports of brick size distributions in synthetic materials, it is reasonable to assume that their distributions are similar to those of natural materials, with standard deviations on the same order of magnitude as the simulation results presented here.

A given collection of random widths then defines a given specimen; each specimen's microstructure (i.e. a specific population sample of bricks) is dictated by a random number seed (see Fig. 1d). This allows for different specimens from the same population mean and standard deviation in brick sizes to be easily generated (similar to what one would observe in two different experimental specimens). Note that in all cases, the virtual specimens include a large enough sampling of bricks such that the specimen's mean brick size and standard deviation were within one percent of the pre-specified population mean and standard deviation, respectively. Thus, each specimen is an effective RVE, in that the statistics describing a given specimen are essentially identical to the bricks' population statistics and independent of specimen size.

\subsection{Virtual Test Procedure}

The virtual specimen was generated using a list of straight line segments and connections that define the outer boundary of the specimen, taken here to be a square of width $W$ as shown in Fig. 1. Note that initially, a specimen was generated with $30 \%$ larger dimensions than the actual specimen, with an initial offset equal to one half of the average brick size at its bottom edge. The actual specimen was then 'stamped' from this larger specimen, such that the effects of the stochastic brick sizes and overlaps will have already been well initiated throughout the entire clipped specimen. Note that as larger values of $\Delta \bar{w} /\langle\bar{w}\rangle$ are introduced, the probability that two nearly aligned interfaces (e.g., defects) occurring within the sampled microstructure tends to increase, as shown in Fig. 1d; this is discussed further in the results section.

An initial horizontal edge crack was inserted at the mid-height of the specimen (with $a_{0}=$ $W / 3$ ) by zeroing out cohesive zones in the cracked region, and displacement boundary conditions were applied at the top and bottom edges corresponding to a state of bending superposed with tension as shown in Fig. 1a. Previous work has illustrated that the toughness inferred from this loading configuration is identical to those inferred from alternative types of loading, verifying the interpretation of the initiation toughness as a specimen-independent property [33].

The equilibrium configuration of brick positions during incremental loading was solved for using 
(a)

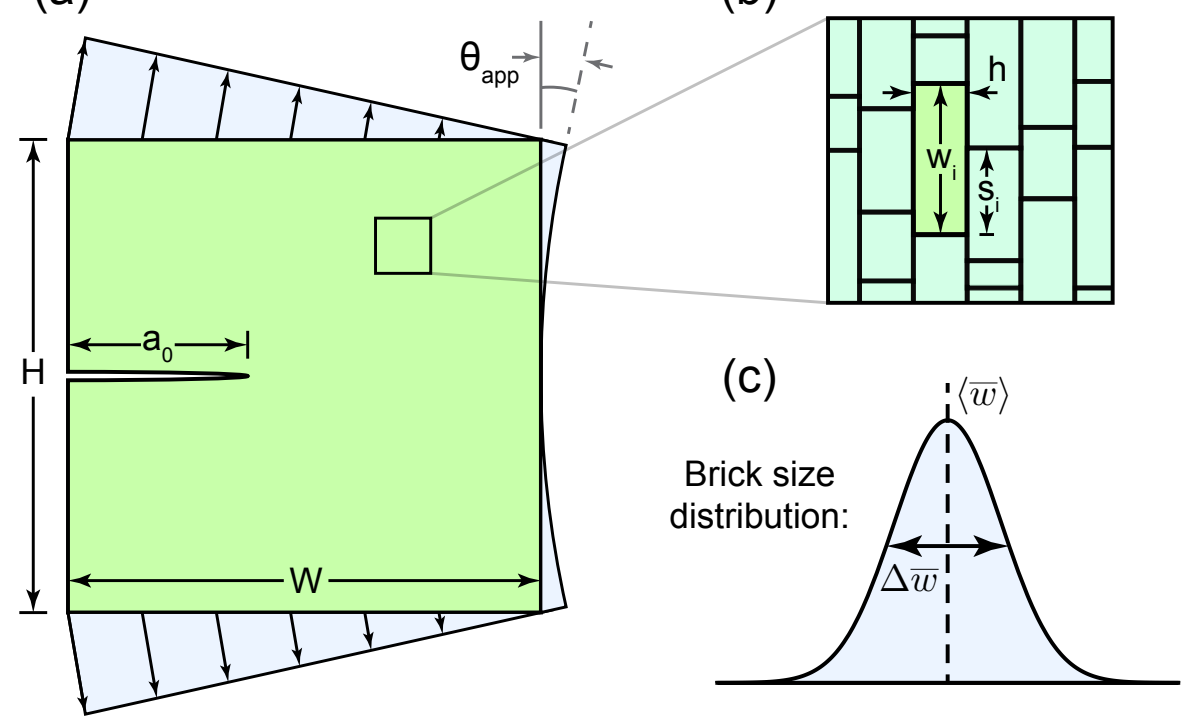

(b) (d) $\Delta \bar{w} /\langle\bar{w}\rangle=.01 \quad \Delta \bar{w} /\langle\bar{w}\rangle=.03 \quad \Delta \bar{w} /\langle\bar{w}\rangle=.10$
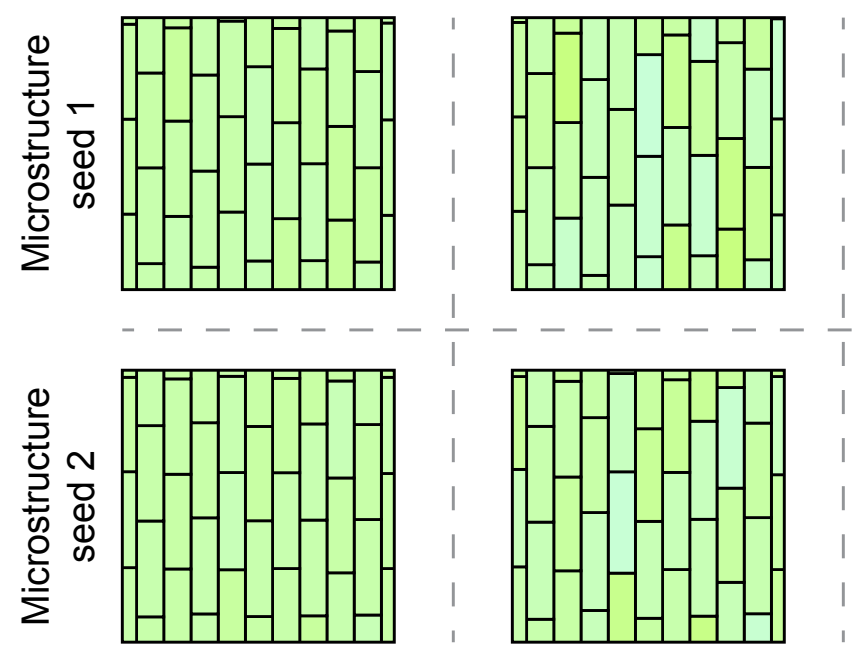

Figure 1: Schematic of (a) macroscopic specimen and loading; (b) brick parameters; (c) probability density function of brick size generation, and (d) example brick layouts for various brick distributions with different population standard deviations $\Delta \bar{w}$ (columns) and population samples (rows), controlled by the microstructure random number seed. Brick colors indicate the size of a given brick relative to the population mean, $\bar{w}_{i} /\langle\bar{w}\rangle$. Nearly aligned horizontal interfaces serve as defects (weak spots), several of which are indicated on $\Delta \bar{w} /\langle\bar{w}\rangle=.1$.

direct search parallel energy minimization (performed on the GPU) via Monte Carlo methods $[33,34]$. As elucidated in [33], this approach is suitable for modeling fracture initiation, for which 

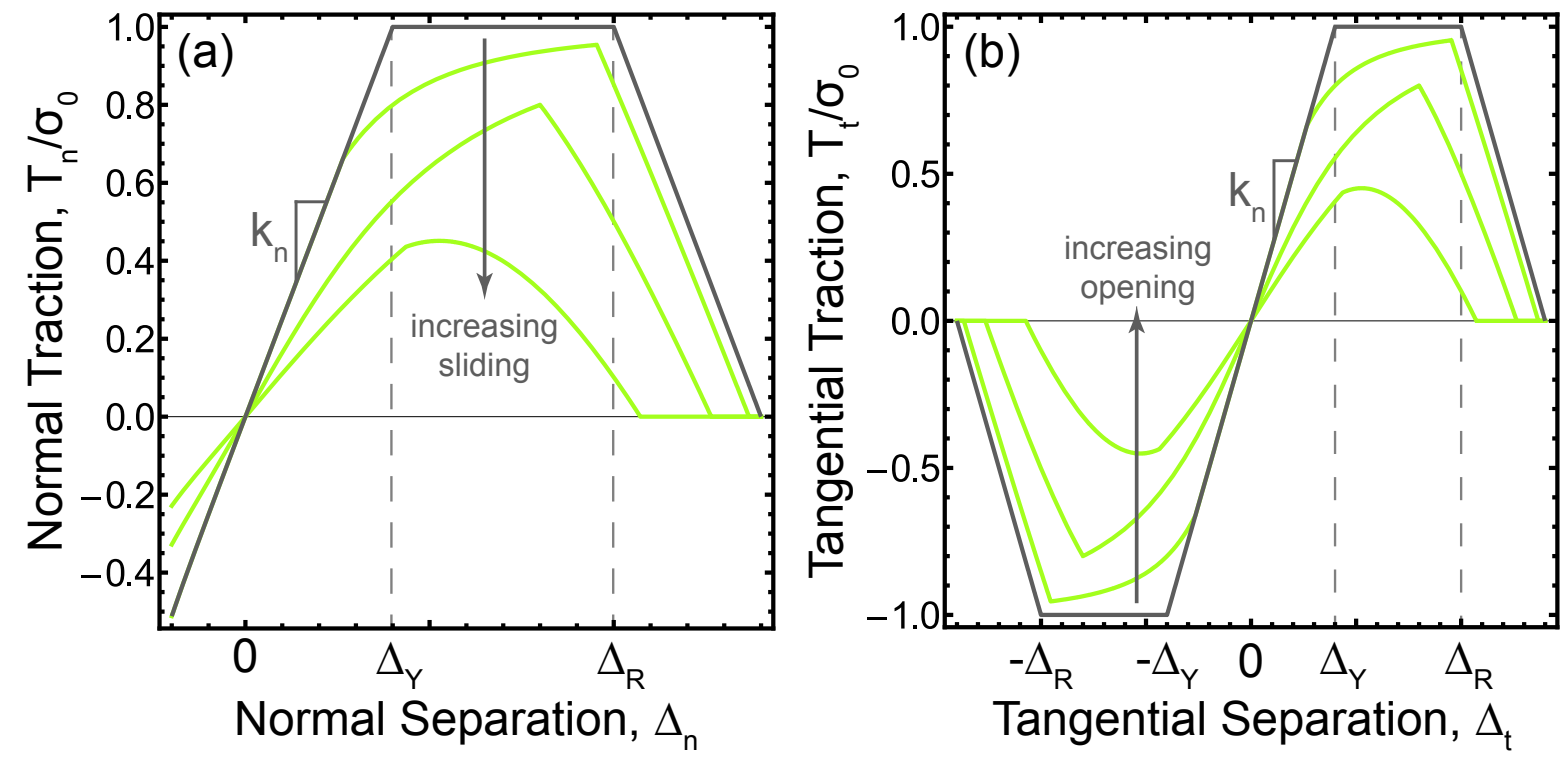

Figure 2: Schematic of the piecewise cohesive law used in analysis for (a) normal and (b) tangential tractions.

unloading is minimal. Strictly speaking, the results of the Monte-carlo minimization itself (i.e. equilibrium states) are generally non-deterministic and depend on the specific minimization path; this path is influenced by the random numbers used to generate candidate brick motions during the minimization step. However, control simulations for a 'perfect' microstructure and different Monte Carlo seeds used in the minimization process show the toughness results are independent of the solution process; these results are discussed in Section 3.1.

The fracture toughness was extracted by the same procedure as in Pro et al [33], which is only briefly summarized here. First, a series of elastic simulations were performed with pre-cracks of increasing lengths, and the total system energy (U) was computed as a function of both loading and pre-crack length. The energy release rate $(G=-\partial U / \partial a)$ was then computed numerically by differentiating the system energy with respect to crack length. Second, a small-scale yielding fracture simulation was performed allowing for interface rupture; the initiation toughness $G_{c}$ was extracted as the energy release rate of the corresponding elastic simulation evaluated at the critical load at which crack initiation was observed. This procedure is only valid when the the energy release rate is dominated by the far field elastic stresses. The requirements among the various length scales for which this is true are outlined in Pro et al. [33].

In order to establish a set of reference values for the composite toughness, a series of control 
simulations were first performed with a 'perfect' microstructure, with all bricks of the exact same size (e.g. $\Delta \bar{w}=0$ and $w_{i} / h=\langle\bar{w}\rangle$ for all brick indices). Stochastic variations were subsequently introduced into the microstructure and the effect of $\Delta \bar{w} /\langle\bar{w}\rangle$ was first studied on the crack driving force $(G)$. Lastly, a series of fracture simulations were performed with both varying brick width standard deviation $\Delta \bar{w}$ and average brick width $\langle\bar{w}\rangle$. For each $\Delta \bar{w} /\langle\bar{w}\rangle$ and $\langle\bar{w}\rangle$ pair, several simulations were run with different specimens (defined by the microstructure seed used to sample the population of brick sizes), and the results for the inferred toughness represent the average over all population samples. Results illustrating the effect of $\Delta \bar{w} /\langle\bar{w}\rangle$ on the average specimen toughness are presented in Section 3.1, and results for the damage and stress ahead of the dominant pre-crack are shown in Section 3.2.

\section{Simulation Results \& Discussion}

\subsection{Energy Release Rates \& Fracture Toughness}

Control simulations were first performed with a set of perfectly stacked bricks with $50 \%$ overlap, all of equal size, for several different brick aspect ratios $\left(\langle\bar{w}\rangle=\bar{w}_{i}=w_{i} / h=1,3.5\right.$, and 5.25 for all brick indices). The effect on the overall crack driving force (normalized appropriately by the work-to-failure of a given interface, $\Gamma_{i}$ ) and inferred toughness was studied for different brick aspect ratios as a function of the Monte Carlo seed. The results confirmed that the influence of the Monte Carlo seed used in the solution method is numerically insignificant. Note that for the control simulations (and for all subsequent calculations), the following specimen sizes (as denoted in Fig. 1 by $\mathrm{H}$ ) were used: $H /\langle w\rangle=316.2,169.0$, and 138.0 for average brick aspect ratios of $\langle w\rangle=1,3.5$, and 5.25 (respectively).

As described in Pro et al. [33], the energy release rate was computed numerically from a series of individual elastic calculations at varying crack lengths. Strictly speaking, for a stochastic microstructure (i.e. a specific set of non-uniform brick arrangements), the energy release rate itself is specimen-dependent. However, it is quite numerically cumbersome to compute a set of separate energy release rates for each specimen; therefore, a set of calculations was next performed to examine the sensitivity of the overall ERR to the microstructure seed.

The simulations revealed that the energy release rate depends only on the average brick size $\langle\bar{w}\rangle$ and the standard deviation $\Delta \bar{w}$ of the population used to create a virtual specimen. That is, 
the energy release rate for a given load level for two different specimens defined from the same population differed in all cases by less than one percent, which is much less than the variability in toughness introduced by even modest values of $\Delta \bar{w}$. Hence, for the remainder of the calculations presented in the subsequent sections, we adopt the approximation $G=f\left(\theta_{a},\langle\bar{w}\rangle, \Delta \bar{w}\right)$. This naturally enables a dramatic reduction in the number of computations, since $G$ does not have to be computed for every single specimen, just for a given set of population statistics.

Note that, because the ERR is scaled directly by the elastic moduli in displacement control (as dictated by dimensionless analysis), it is further implied that the elastic moduli are also functions only of the population statistics $\langle\bar{w}\rangle$ and $\Delta \bar{w}$ (provided enough bricks are sampled). It is worth emphasizing that this confirms the concept that the simulations were conducted for specimens larger than the RVE, since the microstructure's population statistics completely define the macroscopic behavior of the material without explicit specimen dependence.

Although the results for driving force were found to be quite insensitive to the specimen, they were found to be sensitive to the population standard deviation in brick sizes. Fig. 3a shows the effect of $\Delta \bar{w} /\langle\bar{w}\rangle$ on the overall macroscopic crack driving force, $G$, for different average brick aspect ratios. Note that results for only a single population sample are shown in Fig. 3a, but as mentioned previously, these results were highly insensitive to the specific specimen defined from a given $\Delta \bar{w} /\langle\bar{w}\rangle$ and $\langle\bar{w}\rangle$. Again, this implies that the effective elastic properties are functions of only the population mean and standard deviation in brick widths, assuming a large enough specimen is generated. Indeed, the results of Fig. 3a were reproduced to within one percent (not shown here) with a specimen containing twice as many bricks, indicating that enough bricks were sampled.

Fig. 3b shows the averaged initiation toughness $\left\langle G_{c}\right\rangle / \Gamma_{i}$ as a function of $\Delta \bar{w} /\langle\bar{w}\rangle$ for several $\langle\bar{w}\rangle$, computed for twenty specimens with microstructures generated from normal and skew-normal distributions. The error bars in Fig. 3b (as well as $5 \mathrm{a}$ and 6) represent the error due to the discretization in the applied load; the boundary conditions are applied in small increments, and the lower and upper error bounds correspond the simulation frames immediately prior and immediately after (respectively) crack growth has been observed. Note that Fig. 3b also shows results for the 'limit' toughness in the case where the bricks are perfectly stacked, with no deviation in the width $\bar{w}_{i}$ from one brick to the other (i.e., $\Delta \bar{w} /\langle\bar{w}\rangle=0$ ). In this case, the computed toughness values are completely deterministic and therefore are computed from a single specimen. 

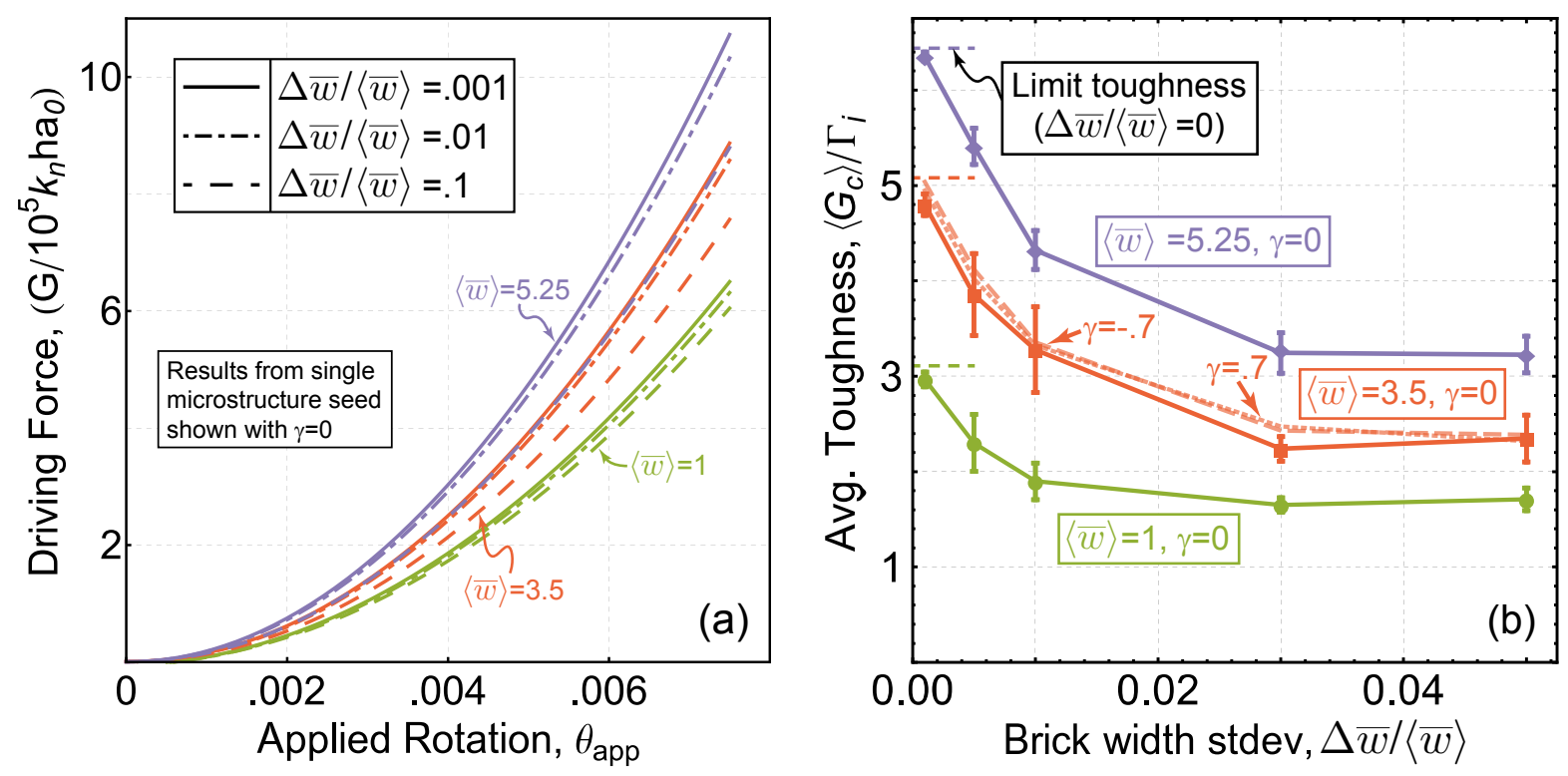

Figure 3: (a) Effect of $\Delta \bar{w} /\langle\bar{w}\rangle$ on the crack driving force, G, for various average brick aspect ratios and a single microstructure seed. (b) Effect of $\Delta \bar{w} /\langle\bar{w}\rangle$ on the average fracture initiation toughness $\left\langle G_{c}\right\rangle$, for average brick aspect ratios of $1,3.5$, and 5.25. Skewness (denoted by $\gamma$ ) introduced only for $\langle\bar{w}\rangle=3.5$. Dashed horizontal lines indicate simulation limit toughness for $\Delta \bar{w} /\langle\bar{w}\rangle=0$. $G_{c}$ averaged over 20 specimens for a given $\Delta \bar{w} /\langle\bar{w}\rangle$. Brick distributions generated using Mersenne Twister pseudorandom number generator.

The results shown in Fig. 3b illustrate the relative tendency of the material to embrittle as the distribution in brick sizes is widened. It is clear from all curves shown in Fig. 3b that all distributions exhibit nearly $50 \%$ reduction in average fracture toughness as larger deviations in brick sizes are introduced into the model, even accounting for the uncertainty involved in the calculation. These results agree well with experimental measurements of hierarchical $A l_{2} O_{3} / P M M A$ composites $[5,6]$. Assuming a bulk toughness $\left(K_{I C}\right)$ of $5 \mathrm{MPa} \sqrt{m}$ and a modulus $(\mathrm{E})$ of $50 \mathrm{GPa}[5,6]$ of the hybrid $\mathrm{Al}_{2} \mathrm{O}_{3} / \mathrm{PMMA}$ composite, one computes the toughness in energetic terms $\left(G_{c}\right)$ to be about $500 \mathrm{~J} / \mathrm{m}^{2}$. The intrinsic toughness of the mortar phase (represented here by $\Gamma_{i}$ ) is more complicated, however, as realistic mortar failure will inevitably involve interacting mechanisms such as bulk mortar deformation, interfacial failure, etc. To first order, we approximate the intrinsic toughness of the mortar as its work to failure in uniaxial tension, but constrained in both transverse directions: $\Gamma_{P M M A}=\frac{1-2 \nu}{1-\nu} \sigma_{Y} \epsilon_{R} t$, where $\sigma_{Y}$ is the uniaxial mortar yield strength, $\epsilon_{R}$ is the full rupture strain, $\mathrm{t}$ is the mortar thickness, and $\nu$ is the mortar Poisson's ratio. Using the following assumed properties: 
$t=2 \mu \mathrm{m}[6], \epsilon_{R}=.6, \sigma_{Y}=50 \mathrm{MPa}[25]$, and $\nu=.35$, one obtains an intrinsic mortar work to failure of about $130 \mathrm{~J} / \mathrm{m}^{2}$ and a relative toughness of $G_{c} / \Gamma_{P M M A}$ of about 3.8, which is slightly higher than the asymptotic values shown in Fig. 3b (approximately 3.2 for $\langle\bar{w}\rangle=5.25$ ). However, it is important to consider that the results in Fig. 3b represent the average fracture toughness from a set of virtual fracture tests, with standard deviations given in Fig. 6. This indeed renders the results of Fig. 3 quite plausible in comparison to experiment, as the measurements of $G_{c} / \Gamma_{P M M A}$ fall well within one standard deviation of the average toughness predicted by Fig. 3. Note that the results shown in Fig. 3b also support the hypothesis that higher volume densities of nearly aligned horizontal interfaces (as indicated by increasing $\Delta \bar{w} /\langle\bar{w}\rangle$ in Fig. 1d) tend to act as defects within the simulated microstructure, and contribute to substantial embrittlement of the bulk material for large $\Delta \bar{w} /\langle\bar{w}\rangle$.

It is also worth mentioning that the toughness computed in Fig. 3 will intuitively depend on the initial brick offset of the referenced 'perfect' material. For example, if the initial offset is zero (analogous to the $0^{\circ}$ case in [33]), introducing stochastics into the simulated microstructure would tend increase the average overlap area, thus providing larger energy dissipation during brick pull-out and effectively raising the toughness of the composite (provided that brick fracture is avoided). In order to limit the scope of the current calculations, the initial offset of all specimens was set at $50 \%$, as noted in Section 2.2. Furthermore, genetic optimization studies with staggered microstructures have suggested that both strength and toughness are optimized at 50\% overlap [37], which further motivates the current parameter study being centered around a processing target of $50 \%$ overlap.

As mentioned previously, the results shown in Fig. 3 are the averaged results for twenty specimens generated from a given $\langle\bar{w}\rangle$ and $\Delta \bar{w}$. It was found that after 20 specimens, the averaged fracture toughness changed by no more than one percent as additional specimens were analyzed. We have rigorously verified this with calculations not presented here by computing both the toughness mean and standard deviations as a function of a sub-set sample size, and found that, even for several different sub-set specimen permutations, the results were always convergent at twenty specimens. Convergence was measured via the slope of a best-fit regression line through the last five curve points in the toughness vs. sample size data. A t-distribution analysis was also performed with the sample mean toughness $\left\langle G_{c}\right\rangle / \Gamma_{i}$ and standard deviation $\Delta G_{c} / \Gamma_{i}$ data. The analysis indicated that the upper and lower bounds on the $95 \%$ confidence interval for the population mean 
toughness were within $10 \%$ of the sample mean toughness for all cases considered here, with most cases being on the order of two to three percent.

We have also verified that small scale yielding conditions are satisfied (as in Pro et al [33]), by doubling the interface ductility $\Delta_{R} / \Delta_{Y}$. This effectively reduces the size of the specimen relative to the damage zone, and it was found that the computed relative fracture toughness $\left\langle G_{c}\right\rangle / \Gamma_{i}$ (again averaged over twenty specimens) does not change by more than a very small percentage upon doubling the interface ductility. Note that this also verifies that a sufficient sampling of bricks was taken in the damage zone ahead of the crack tip, as increasing the interface ductility effectively increases the brick resolution within the damage zone.

\subsection{Damage Zone and Stresses}

Fig. 4a shows color contours of the damage distribution just prior to crack initiation (for a single specimen, or microstructure seed) for several different values of $\Delta \bar{w} /\langle\bar{w}\rangle$, with $\langle\bar{w}\rangle$ held at 3.5. Note that the damage zone is defined by the set of bricks whose adjacent average interface opening displacement has exceeded its critical value [33]. The average damage zone size immediately prior to fracture initiation is also shown in Fig. 5a as a function of both $\Delta \bar{w} /\langle\bar{w}\rangle$ and $\langle\bar{w}\rangle$. The limit damage zone is identified as the number of bricks in the damage zone for the case where no brick variation is present $(\Delta \bar{w} / \bar{w}=0)$. Results for skew normal distributions are also included in Fig. 5a.

It is clear from Figs. 4-5 that the average size (in bricks) of the damage zone just prior to crack initiation is substantially reduced upon introducing stochastic microstructures. Moreover, the data in Fig. 5a correlates well with the toughness data in Fig. 3b; as the size of the damage zone is decreased, the corresponding average fracture toughness decreases. This verifies that the process zone is the main contributor to the overall toughness in the simulations, as expected from previous analysis of the toughening mechanisms in brick and mortar composites [29]. Similarly, the effect of skewness on the damage zone size seems relatively small (but slightly beneficial) as deduced from $5 \mathrm{a}$, provided that $\Delta \bar{w} /\langle\bar{w}\rangle$ is held constant. While it is difficult to discern given the error involved in the calculations, note that Figs. 3b and 5a also mildly suggest that, for $\Delta \bar{w} /\langle\bar{w}\rangle>.03$, stochastics in the microstructure could actually introduce a slight benefit in toughness.

Figs. $4 \mathrm{~b}$ and $5 \mathrm{~b}$ show the vertical brick stresses $\sigma_{22}$ in the vicinity of the crack tip for different values of $\Delta \bar{w} /\langle\bar{w}\rangle$, both as contours and linear plots (respectively). The resulting equilibrium stress 

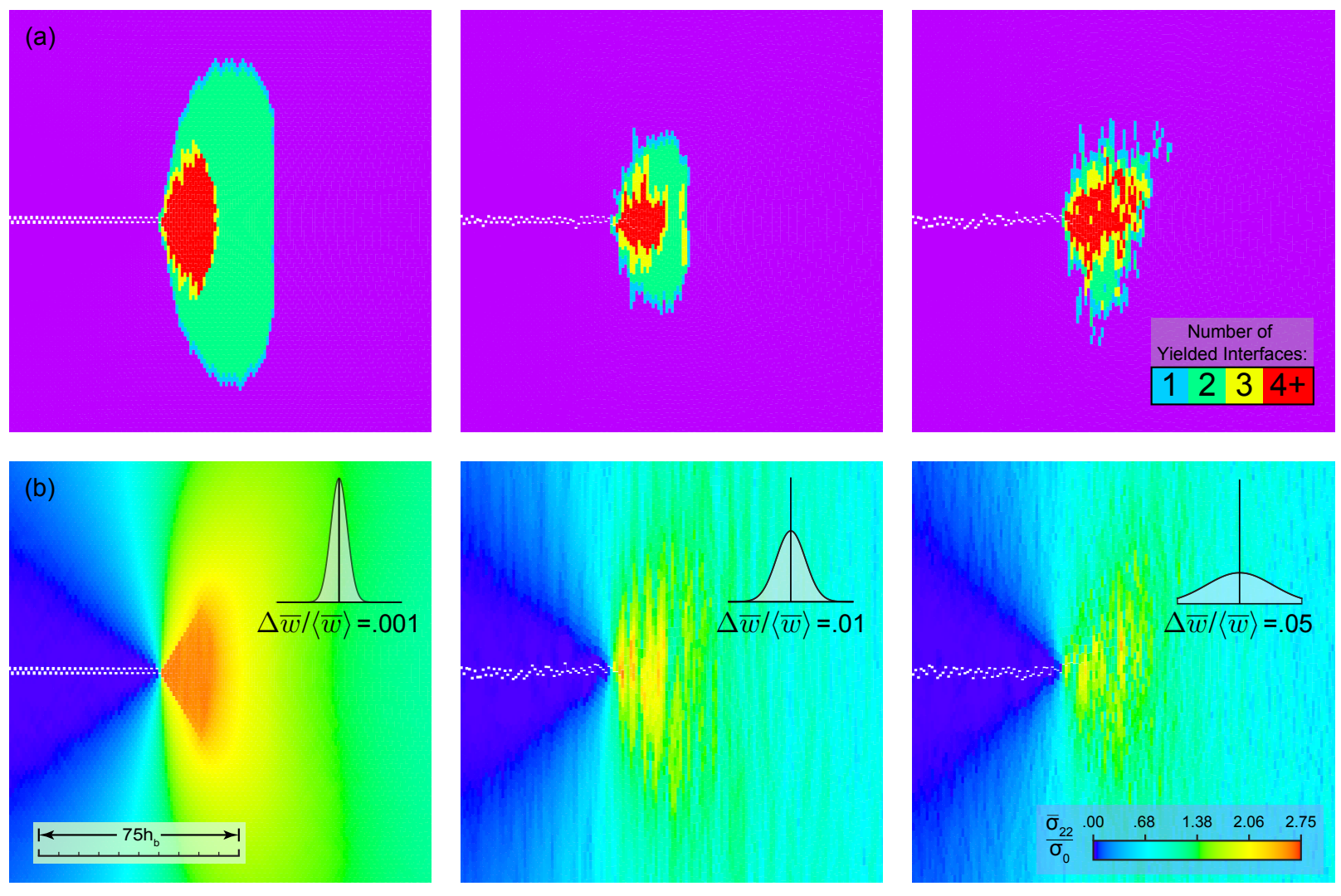

Figure 4: (a) Damage zone contours ahead of dominant pre-crack for $\langle\bar{w}\rangle=3.5$ and various values of $\Delta \bar{w} /\langle\bar{w}\rangle$. Bricks colored according to the number of adjacent average interface opening has exceed the rupture displacement of the cohesive law. (b) Vertical brick stresses $\sigma_{22}$ ahead of initial pre-crack for several values of $\Delta \bar{w} /\langle\bar{w}\rangle$. Stresses represent the average volumetric brick stresses and are scaled by the cohesive strength of the adjacent interfaces. Frames are taken immediately prior to fracture and represent a single specimen for each value of $\Delta \bar{w} /\langle\bar{w}\rangle$. Each close up displayed at approximately 3.6x linear magnification relative to entire simulation specimen.

patterns are rather complex; large variations in stress magnitude become present as randomness is introduced into the brick sizes. Moreover, as $\Delta \bar{w} /\langle\bar{w}\rangle$ increases, the relative stress oscillation amplitude from brick to brick tends to increase. Note that this makes it difficult to identify the composite strength from crack tip fields, as the stresses within the damage zone do not remain clearly constant as in the case with $\Delta \bar{w} /\langle\bar{w}\rangle \approx 0$. However, it is clear from Fig. $4 \mathrm{~b}$ and $5 \mathrm{~b}$ that the average normalized peak stress within the damage zone, which directly scales the normalized composite strength, is reduced. This suggests that a lower composite strength (in addition to toughness) is also realized when introducing randomness into the brick sizes, and the well-known 

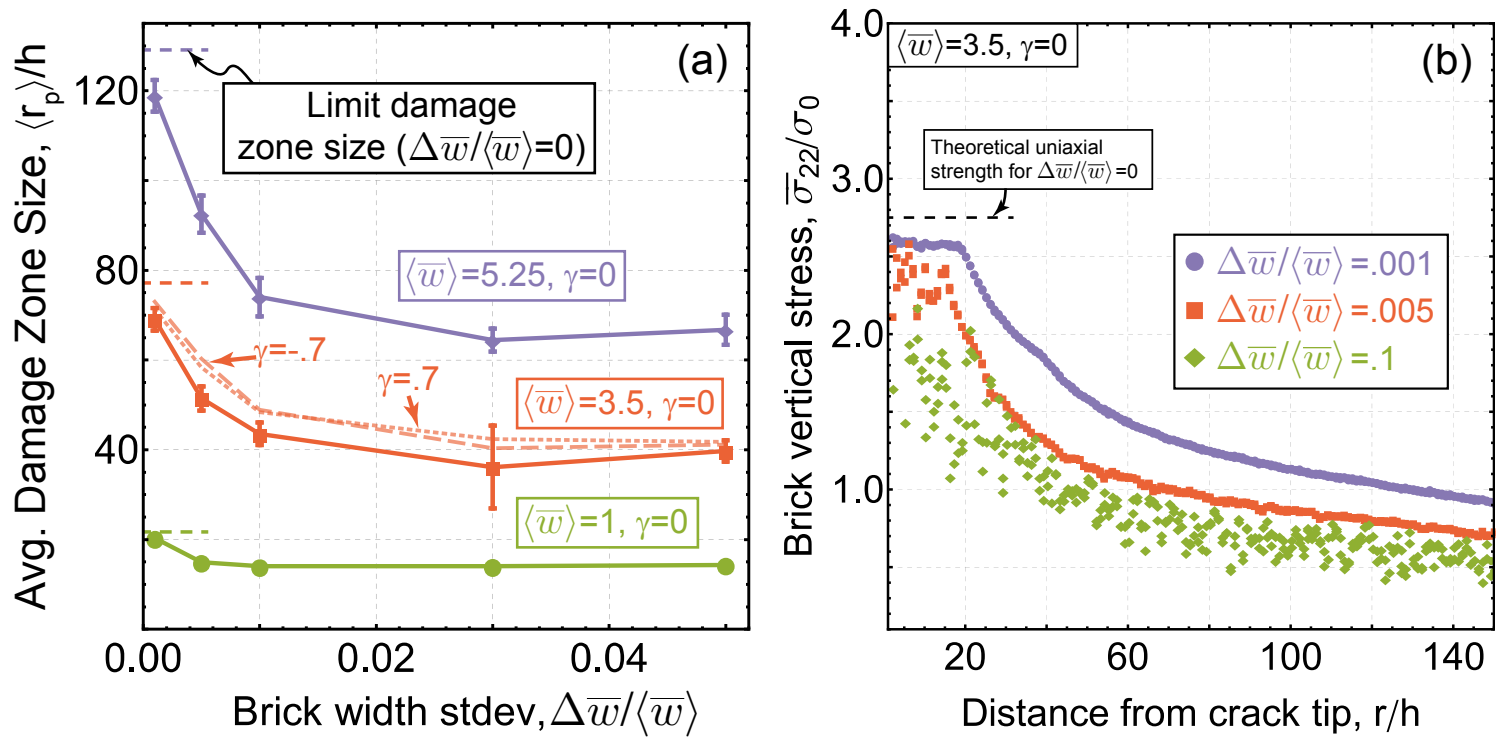

Figure 5: (a) Average damage zone size as a function $\Delta \bar{w} /\langle\bar{w}\rangle$, for various brick aspect ratios, (including positive \& negative skew-normal distributions). Damage zone size is measured immediately prior to fracture initiation, and is defined as $\left\langle r_{p}\right\rangle / h=\sqrt{N_{p}\langle\bar{w}\rangle / h}$, where $N_{p}$ is the number of bricks in the damage zone. (b) Stress distribution directly ahead of crack of crack tip for $\langle\bar{w}\rangle=3.5$ and $\Delta \bar{w} /\langle\bar{w}\rangle=.001,005$, and .1. Theoretical strength predicted by uniaxial model of Bekah et al [35] (with $\mathrm{k}=1$ ) indicated by horizontal dashed line.

strength enhancement due to shear transfer [25, 26, 31, 37, 38] between bricks is diminished as uneven and random overlaps are distributed throughout the composite. This trend is also in good agreement with the strength simulation results of Bekah et al [35], which also implemented stochastic microstructures.

As Fig. 3b shows the average toughness from a subset of fracture tests, Fig. 6 then shows the standard deviation in toughness from that same subset of fracture tests (denoted by $\Delta G_{c} / \Gamma_{i}$ ). The results are similarly presented as a function of $\Delta \bar{w} /\langle\bar{w}\rangle$ for different values of $\langle\bar{w}\rangle$. Several features of Fig. 6 are immediately apparent, and when combined with the results presented in previous figures illustrate several important engineering tradeoffs. First of all, $\Delta G_{c} / \Gamma_{i}$ tends to decrease monotonically in the range $\Delta \bar{w} /\langle\bar{w}\rangle \approx .01-.03$ for all simulation results shown in Fig. 6 . Combined with the results shown in Fig. 3b, this implies that a tradeoff exists between average toughness magnitude and variability in toughness (indicated by its standard deviation $\Delta G_{c} / \Gamma_{i}$ ) in the range $\Delta \bar{w} /\langle\bar{w}\rangle \approx .01-.03$. In other words, decreasing $\Delta \bar{w} /\langle\bar{w}\rangle$ in this region increases the toughness but also increases the variability in toughness, which has substantial implications in 


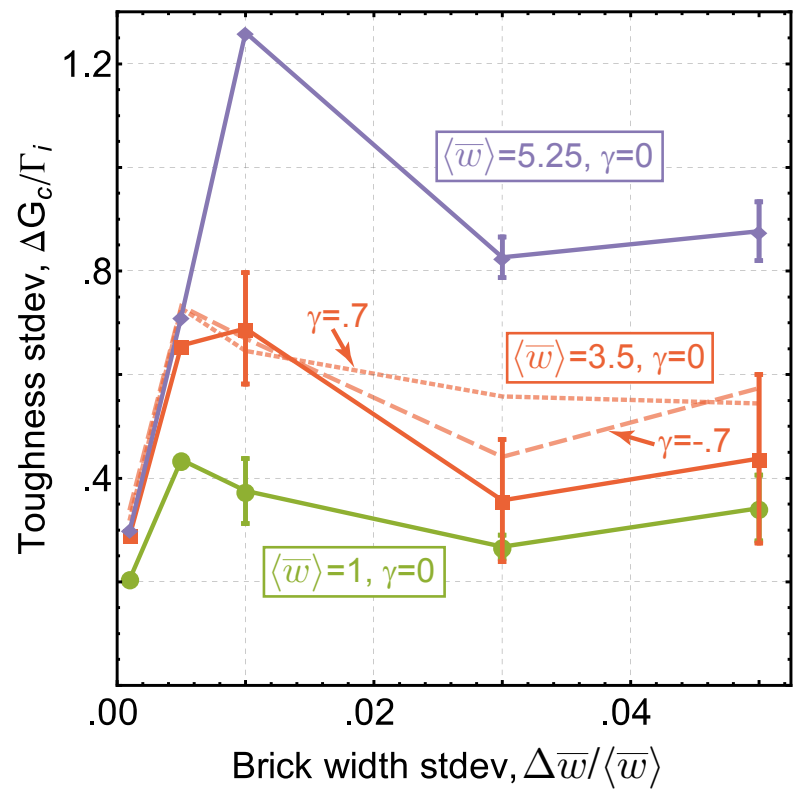

Figure 6: Standard deviation in toughness $\Delta G_{c}$ (relative to the intrinsic interface toughness $\Gamma_{i}$ ), as a function of $\Delta \bar{w} /\langle\bar{w}\rangle$, for several different average aspect ratios and skewness parameters.

design and fabrication of these complex hierarchical composites. For values of $\Delta \bar{w} /\langle\bar{w}\rangle>.03$, the error-to-measurement ratio is too high to draw meaningful conclusions about the trends in $\Delta G_{c} / \Gamma_{i}$.

It is important to note that, while clear trade-offs exist in the region $\Delta \bar{w} /\langle\bar{w}\rangle \approx .01-.03$, the results in the range $\Delta \bar{w} /\langle\bar{w}\rangle \approx 0-.01$ tend to suggest the contrary. In this region, decreasing $\Delta \bar{w} /\langle\bar{w}\rangle$ clearly decreases the variability in toughness while also increasing the average toughness, both desirable traits that seem to be obtained simultaneously. Effectively, as $\Delta \bar{w} /\langle\bar{w}\rangle$ decreases in this region, the results are brought closer to deterministic failure, as one would expect with a perfectly architectured composite. It is important, however, to also consider the significant increases in cost and manufacturing complexity as $\Delta \bar{w} /\langle\bar{w}\rangle$ is brought closer to zero.

Fig. 6 also indicates that increases in average aspect ratio $\langle\bar{w}\rangle$ tend to also increase the variability in the computed toughness, as illustrated for the range of brick aspect ratios presented. Again, this illustrates another important tradeoff when combined with the implications of Fig. 3b; as the brick aspect ratio increases, the average toughness increases, but so does the variability in toughness. Moreover, as illustrated in micro-mechanical models [25], increases in brick aspect ratio tend to increase the likelihood of catastrophic failure via brick fracture due to an amplified stress state and their limited strength. In this case, all toughness due to microstructure is lost, which is a well 
known tradeoff between toughness and strength in these composites [39].

The results presented in this section illustrate the relative engineering tradeoffs between composite properties and the allowable deviation in brick width $\Delta \bar{w} /\langle\bar{w}\rangle$. The simulation results quantify the specific sensitivity of the composite properties to the statistical properties of the distribution in brick sizes. As illustrated in Figs. 3-5, a substantial (30-50\%) drop in toughness and damage zone size is exhibited even with just a one percent deviation in brick size, as well as a significant dispersion of the vertical brick stresses.

\section{Concluding Remarks}

The results presented in this paper show a powerful application of the method presented by Pro et al. [33] and Lim et al. [34], illustrating general capabilities to simulate large specimens that contain distributions in brick sizes; further, the speed enables one to simulate enough fracture tests to obtain meaningful statistics on the macroscopic fracture properties. The key results and their implications are outlined below:

- Variability in brick sizes decreases the effective elastic properties of the composite, which directly scale the macroscopic energy release rate to drive growth of a dominant flaw. The ERR was also found to be insensitive to specific population sample (controlled by the microstructure seed), allowing for a drastic reduction in the total computational cost.

- Average composite toughness $\left\langle G_{c}\right\rangle / \Gamma_{i}$ drops substantially as larger variations $(\Delta \bar{w} /\langle\bar{w}\rangle)$ are introduced, on the order of $50 \%$ with $\Delta \bar{w} /\langle\bar{w}\rangle \simeq 0.03$. The reduction in toughness is also accompanied by a reduction in the damage zone size, as suggested by models [29]. The results also confirm the well known benefit of aspect ratio on the composite toughness $\left\langle G_{c}\right\rangle / \Gamma_{i}[29]$.

- $\Delta G_{c} / \Gamma_{i}$ is maximized at around $\Delta \bar{w} /\langle\bar{w}\rangle=.01$, indicating that for $\Delta \bar{w} /\langle\bar{w}\rangle$ larger than .01, a trade-off exists between toughness $\left\langle G_{c}\right\rangle / \Gamma_{i}$ and the variability of the toughness $\Delta G_{c} / \Gamma_{i}$; Higher toughness cannot be achieved without also increasing the variability in toughness. For $\Delta \bar{w} /\langle\bar{w}\rangle<.01$, no trade-off between toughness and its variability exist.

- Large values of $\Delta \bar{w} /\langle\bar{w}\rangle$ tend to disperse stresses ahead of the crack tip, thus leading to (on average) lower stresses in the damage zone directly ahead of the dominant flaw. This implies composite failure strength is also reduced substantially for large $\Delta \bar{w} /\langle\bar{w}\rangle$. 


\section{Acknowledgements:}

This research was financially supported through grant DMR-1105672 from the National Science foundation, grant W911NF-09-0001 through the Institute for Collaborative Biotechnologies from the U.S. Army Research office, and grant DOE/DE-SC0008975 from the Department of Energy. Additional computational resources were provided by the Center for Scientific Computing at the CNSI and MRL: an NSF MRSEC (DMR-1121053) and NSF CNS-0960316. The content and information presented does not reflect the position or policy of the U.S. government, and no official endorsement should be inferred or implied. 


\section{References:}

[1] Z. Zhang, B. Liu, Y. Huang, K. Hwang, and H. Gao, "Mechanical properties of unidirectional nanocomposites with non-uniformly or randomly staggered platelet distribution," Journal of the Mechanics and Physics of Solids, vol. 58, no. 10, pp. 1646-1660, 2010.

[2] H. Lei, Z. Zhang, and B. Liu, "Effect of fiber arrangement on mechanical properties of short fiber reinforced composites," Composites Science and Technology, vol. 72, no. 4, pp. 506-514, 2012.

[3] Z. Zhang, Y.-W. Zhang, and H. Gao, "On optimal hierarchy of load-bearing biological materials," Proceedings of the Royal Society B: Biological Sciences, p. rspb20101093, 2010.

[4] Z. Tang, N. A. Kotov, S. Magonov, and B. Ozturk, "Nanostructured artificial nacre," Nature materials, vol. 2, no. 6, pp. 413-418, 2003.

[5] M. E. Launey, E. Munch, D. Alsem, H. Barth, E. Saiz, A. P. Tomsia, and R. O. Ritchie, "Designing highly toughened hybrid composites through nature-inspired hierarchical complexity," Acta Materialia, vol. 57, no. 10, pp. 2919-2932, 2009.

[6] E. Munch, M. E. Launey, D. H. Alsem, E. Saiz, A. P. Tomsia, and R. O. Ritchie, "Tough, bio-inspired hybrid materials," Science, vol. 322, no. 5907, pp. 1516-1520, 2008.

[7] F. Barthelat and D. Zhu, "A novel biomimetic material duplicating the structure and mechanics of natural nacre," Journal of Materials Research, vol. 26, no. 10, pp. 1203-1215, 2011.

[8] A. Walther, I. Bjurhager, J.-M. Malho, J. Pere, J. Ruokolainen, L. A. Berglund, and O. Ikkala, "Large-area, lightweight and thick biomimetic composites with superior material properties via fast, economic, and green pathways," Nano letters, vol. 10, no. 8, pp. 2742-2748, 2010.

[9] S. Deville, E. Saiz, R. K. Nalla, and A. P. Tomsia, "Freezing as a path to build complex composites," Science, vol. 311, no. 5760, pp. 515-518, 2006.

[10] F. Barthelat and H. Espinosa, "An experimental investigation of deformation and fracture of nacre-mother of pearl," Experimental mechanics, vol. 47, no. 3, pp. 311-324, 2007. 
[11] Y. Shao, H.-P. Zhao, and X.-Q. Feng, "On flaw tolerance of nacre: a theoretical study," Journal of The Royal Society Interface, vol. 11, no. 92, p. 20131016, 2014.

[12] F. Barthelat, "Nacre from mollusk shells: a model for high-performance structural materials," Bioinspiration ES Biomimetics, vol. 5, no. 3, p. 035001, 2010.

[13] K. Gries, R. Kröger, C. Kübel, M. Fritz, and A. Rosenauer, "Investigations of voids in the aragonite platelets of nacre," Acta biomaterialia, vol. 5, no. 8, pp. 3038-3044, 2009.

[14] T. Verho, M. Karesoja, P. Das, L. Martikainen, R. Lund, A. Alegría, A. Walther, and O. Ikkala, "Hydration and dynamic state of nanoconfined polymer layers govern toughness in nacremimetic nanocomposites," Advanced Materials, vol. 25, no. 36, pp. 5055-5059, 2013.

[15] Z. Xie and H. Yao, "Crack deflection and flaw tolerance in" brick-and-mortar" structured composites," International Journal of Applied Mechanics, vol. 6, no. 02, p. 1450017, 2014.

[16] H. Gao, B. Ji, M. J. Buehler, and H. Yao, "Flaw tolerant bulk and surface nanostructures of biological systems," Mech. Chem. Biosyst, vol. 1, no. 1, pp. 37-52, 2004.

[17] L. J. Bonderer, A. R. Studart, and L. J. Gauckler, "Bioinspired design and assembly of platelet reinforced polymer films," Science, vol. 319, no. 5866, pp. 1069-1073, 2008.

[18] F. Barthelat, H. Tang, P. Zavattieri, C.-M. Li, and H. Espinosa, "On the mechanics of motherof-pearl: a key feature in the material hierarchical structure," Journal of the Mechanics and Physics of Solids, vol. 55, no. 2, pp. 306-337, 2007.

[19] F. Song, A. Soh, and Y. Bai, "Structural and mechanical properties of the organic matrix layers of nacre," Biomaterials, vol. 24, no. 20, pp. 3623-3631, 2003.

[20] F. Song, X. Zhang, and Y. Bai, "Microstructure and characteristics in the organic matrix layers of nacre," Journal of materials research, vol. 17, no. 07, pp. 1567-1570, 2002.

[21] R. Wang, Z. Suo, A. Evans, N. Yao, and I. Aksay, "Deformation mechanisms in nacre," Journal of Materials Research, vol. 16, no. 09, pp. 2485-2493, 2001.

[22] A. Jackson, J. Vincent, and R. Turner, "The mechanical design of nacre," Proceedings of the Royal society of London. Series B. Biological sciences, vol. 234, no. 1277, pp. 415-440, 1988. 
[23] A. Jackson, J. Vincent, and R. Turner, "Comparison of nacre with other ceramic composites," Journal of Materials Science, vol. 25, no. 7, pp. 3173-3178, 1990.

[24] A. Jackson, J. Vincent, and R. Turner, "A physical model of nacre," Composites Science and Technology, vol. 36, no. 3, pp. 255-266, 1989.

[25] M. R. Begley, N. R. Philips, B. G. Compton, D. V. Wilbrink, R. O. Ritchie, and M. Utz, "Micromechanical models to guide the development of synthetic brick and mortar composites," Journal of the Mechanics and Physics of Solids, vol. 60, no. 8, pp. 1545-1560, 2012.

[26] D. V. Wilbrink, M. Utz, R. O. Ritchie, and M. R. Begley, "Scaling of strength and ductility in bioinspired brick and mortar composites," Applied Physics Letters, vol. 97, no. 19, p. 193701, 2010.

[27] S. Kotha, Y. Li, and N. Guzelsu, "Micromechanical model of nacre tested in tension," Journal of materials science, vol. 36, no. 8, 2001.

[28] K. Bertoldi, D. Bigoni, and W. Drugan, "Nacre: an orthotropic and bimodular elastic material," Composites Science and Technology, vol. 68, no. 6, pp. 1363-1375, 2008.

[29] F. Barthelat and R. iei, "Toughness amplification in natural composites," Journal of the Mechanics and Physics of Solids, vol. 59, no. 4, pp. 829-840, 2011.

[30] M. Genet, G. Couégnat, A. P. Tomsia, and R. O. Ritchie, "Scaling strength distributions in quasi-brittle materials from micro-to macro-scales: A computational approach to modeling nature-inspired structural ceramics," Journal of the Mechanics and Physics of Solids, vol. 68, pp. 93-106, 2014.

[31] F. Barthelat, "Designing nacre-like materials for simultaneous stiffness, strength and toughness: Optimum materials, composition, microstructure and size," Journal of the Mechanics and Physics of Solids, vol. 73, pp. 22-37, 2014.

[32] F. Barthelat and M. Mirkhalaf, "The quest for stiff, strong and tough hybrid materials: an exhaustive exploration," Journal of The Royal Society Interface, vol. 10, no. 89, p. 20130711, 2013. 
[33] J. W. Pro, R. K. Lim, L. R. Petzold, M. Utz, and M. R. Begley, "Gpu-based simulations of fracture in idealized brick and mortar composites," Journal of the Mechanics and Physics of Solids, vol. 80, pp. 68-85, 2015.

[34] R. K. Lim, J. W. Pro, M. R. Begley, M. Utz, and L. R. Petzold, "High-performance simulation of fracture in idealized brick and mortarcomposites using adaptive monte carlo minimization on the gpu," International Journal of High Performance Computing Applications, p. 1094342015593395, 2015.

[35] S. Bekah, R. Rabiei, and F. Barthelat, "The micromechanics of biological and biomimetic staggered composites," Journal of Bionic Engineering, vol. 9, no. 4, pp. 446-456, 2012.

[36] M. Matsumoto and T. Nishimura, "Dynamic creation of pseudorandom number generators," Monte Carlo and Quasi-Monte Carlo Methods, vol. 2000, pp. 56-69, 1998.

[37] H. Gao, "Application of fracture mechanics concepts to hierarchical biomechanics of bone and bone-like materials," International Journal of Fracture, vol. 138, no. 1-4, pp. 101-137, 2006.

[38] S. Bekah, R. Rabiei, and F. Barthelat, "Structure, scaling, and performance of natural microand nanocomposites," BioNanoScience, vol. 1, no. 1-2, pp. 53-61, 2011.

[39] R. O. Ritchie, "The conflicts between strength and toughness," Nature materials, vol. 10, no. 11, pp. 817-822, 2011. 